\section{Validation of the Food-Linked Virtual Response task}

\author{
Elizabeth A Pascoe', Laura Smart Richman² \\ and Daniel Kort²
}

Journal of Health Psychology 1-9

(c) The Author(s) 2015

Reprints and permissions: sagepub.co.uk/journalsPermissions.nav DOI: $10.1177 / 1359105315595452$ hpq.sagepub.com

(S)SAGE

\begin{abstract}
This research validates a computerized dietary selection task (Food-Linked Virtual Response or FLVR) for use in studies of food consumption. In two studies, FLVR task responses were compared with measures of health consciousness, mood, body mass index, personality, cognitive restraint toward food, and actual food selections from a buffet table. The FLVR task was associated with variables which typically predict healthy decision-making and was unrelated to mood or body mass index. Furthermore, the FLVR task predicted participants' unhealthy selections from the buffet, but not overall amount of food. The FLVR task is an inexpensive, valid, and easily administered option for assessing momentary dietary decisions.
\end{abstract}

\title{
Keywords
}

eating behavior, health behavior, methodology, quantitative methods, validation

Because dietary habits are consistently associated with health and health risk, much research focuses on how individuals make food-related decisions. Typically, researchers study diet by asking participants to recall information about recently consumed food and drink (e.g. Lee et al., 2011). Alternately, participants may report their consumption likelihood when experiencing certain circumstances, such as variations in emotional state (Koball et al., 2012). Recall bias can be a concern, although it may be attenuated by the use of a food diary (e.g. Brogan and Hevey, 2013). However, neither option allows for the determination of causal effects of dietary decision-making.

Causal effects can be assessed using experimental paradigms in which participants' momentary food choices are monitored. Typically, this is accomplished by providing buffet tables and observing participants' interaction with the supplied edibles, such as weighing bowls before and after each participant or observing choice as it happens (Robinson et al., 2014). However, providing and maintaining a buffet of appetizing food requires much effort and expense.

One solution to such limitations is to develop an inexpensive, easily implemented measure to approximate the dietary decisions individuals would make in lieu of providing actual food. Pascoe and Richman (2010) investigated the effect of discrimination on subsequent foodrelated decision-making using such a task. Participants were required to make a series of food-related selections via computer, with the

\footnotetext{
'University of North Carolina at Asheville, USA 2Duke University, USA

Corresponding author:

Elizabeth A Pascoe, Department of Psychology, University of North Carolina at Asheville, I University Heights, CPO 1630, Asheville, NC 8804-8508, USA.

Email: epascoe@unca.edu
} 
instructions to select what they would most like to eat at that moment. The aim of the task was to determine how often participants made unhealthy selections when faced with healthy and unhealthy options simultaneously.

The following studies were designed to refine and validate the computerized FoodLinked Virtual Response (FLVR) task as used by Pascoe and Richman so that it can be used in future research studying dietary decision-making. In Study 1, participants viewed food images, choosing between two food-picture options using the computerized FLVR task. In order to determine convergent and divergent validity, participants also reported level of health consciousness, cognitive restraint with respect to food, mood, and completed the Big 5 personality inventory. In Study 2, participants were able to select actual food from a buffetlike presentation shortly after completing the FLVR task. The FLVR task was expected to correlate with variables measuring preference for healthy living (e.g. health consciousness and dietary restraint). The FLVR task was also expected to be related to the personality dimension of conscientiousness, high levels of which correlate positively with better physical health and lifespan (Kern and Friedman, 2008), and a variety of healthy behaviors, such as diet and exercise (Bogg and Roberts, 2004). Positive and negative mood were also addressed, although were not anticipated to relate to the FLVR task. Body mass index (BMI) and the personality variables of neuroticism, openness, agreeableness, and extraversion were not assumed to correlate with FLVR task scores. Finally, unhealthy selections via computer were expected to predict an increased tendency to select unhealthy options from the buffet.

\section{Study I}

This study assessed the construct validity for the FLVR task. Low health consciousness, low dietary restraint, low exercise levels, and low conscientiousness were expected to correlate with more unhealthy FLVR task selections. Positive and negative mood, BMI, and the remaining personality variables were expected to be unrelated to FLVR task scores.

\section{Method}

Participants. In total, 106 community members (56\% female; 61.4\% White, 7.5\% Black, 7.5\% Latino, $15.1 \%$ Asian, 1.9\% Native American, and $6.6 \%$ other; mean age $=26.56$ years, standard deviation $(S D)=8.49$ ) were recruited in an urban area of the Southeastern United States.

Procedure. Following recruitment and informed consent, participants completed several questionnaires on computers provided by the researchers. Participants reported demographic information including race, gender, height, weight, age, and vegetarian/omnivore status. Participants were debriefed and compensated for their participation.

FLVR task. The FLVR task is a computerized food-based decision task designed to approximate the type of food selections an individual might make if food choices were readily available. The FLVR task was developed by selecting pictures of potentially healthy and unhealthy food and beverage items. Four independent raters assessed the healthiness of each item on a 10 -point scale ranging from -5 (very unhealthy) to +5 (very healthy). Pictures receiving an average absolute value score of 4 or 5 were retained for use as FLVR task stimuli. In total, 99 pairs of food/beverage stimuli were created by matching pictures for valence of health quality using the average ratings from the 10-point scale described above. Food pairs were also matched by food type (e.g. breakfast, snack, dessert, and beverage) and approximate amount of food depicted. In total, 49 pairs contrasted healthy with unhealthy choices (e.g. grilled chicken vs fried chicken) (heretofore referred to as "target" trials), ${ }^{1} 25$ contrasted two healthy choices (e.g. celery vs banana) (healthy trials), and 25 contrasted two unhealthy choices (e.g. ice cream vs chocolate cake) (unhealthy trials).

To engage in the task, participants were presented a food pair on a computer screen and 
instructed to "as quickly as possible, choose the option, which, if it were presented to you in real life at this moment, you would be most likely to choose to eat or drink." Choices were indicated by pressing designated letter keys that corresponded to each side of the screen. Stimuli were presented in random order. The task was scored by tallying the number of unhealthy choices made in the 49 target trials $(\alpha$ target trials $=.90)$.

Godin Leisure-Time Exercise Questionnaire. The Godin Leisure-Time Exercise Questionnaire (GLTEQ) measures exercise habits (Godin and Shepherd, 1997). Participants reported how many times per week they engaged in exercise for greater than 15 minutes during their free time. Participants also rated the frequency with which they engaged in sweat-producing activity.

Health Consciousness Scale. The Health Consciousness Scale (HCS) measures dispositional attitudes toward one's health (Gould, 1988) (e.g. "I'm usually aware of my health"). Responses were summed to generate a total health consciousness score $(\alpha=.94)$.

Positive and Negative Affect Scale. The Positive and Negative Affect Scale (PANAS) measures affective/emotional predisposition (Watson et al., 1988). Positive affect $(\alpha=.92)$ and negative affect $(\alpha=.81)$ sub-scores were generated by summing the 20 positive and negative items.

Big Five Inventory-10. The Big Five Inventory-10 measures personality (Rammstedt and John, 2007). Openness ( $\alpha=.24)$, conscientiousness $(\alpha=.51)$, extraversion $(\alpha=.64)$, agreeableness $(\alpha=.073)$, and neuroticism $(\alpha=.58)$ sub-scores were generated by summing the two items corresponding to each trait.

Three-Factor Eating Questionnaire. The ThreeFactor Eating Questionnaire (TFEQ) measures cognitive restraint of eating, disinhibition, and hunger (Stunkard and Messick, 1985). This study included only cognitive restraint of eating. Items were re-scored according to the instrument's instructions and summed to create an index of cognitive restraint in eating behavior $(\alpha=.73)$.

BMI. BMI was calculated from self-reported height and weight measurements using the standard formula for pounds and inches.

\section{Results and discussion}

In general, participants made fewer unhealthy than healthy selections on target trials of the FLVR task $(M=18.91, S D=9.63) .{ }^{2}$ Independent sample $t$-tests examined whether performance on the FLVR task differed by gender and vegetarian status. ${ }^{3}$ Men and women scored similarly on the FLVR task $(t(102)=1.65, p>.05)$ as did vegetarians and non-vegetarians $(t(102)=-.88$, $p>.05$ ). One-way analysis of variance (ANOVA) determined that FLVR task scores varied between racial groups $(F(5,98)=2.51, p<.04)$ although Tukey's post hoc tests revealed no specific differences. FLVR task was negatively correlated with age $(r=-.33)$, with younger individuals selecting unhealthier options.

As expected, FLVR task scores correlated negatively with cognitive restraint in eating $(r=-.28)$, such that individuals reporting more habitual thought about controlling diet were less likely to select unhealthy options on FLVR task target trials (see Table 1). ${ }^{4}$ Similarly, health consciousness was negatively correlated with the FLVR task $(r=-.20)$. Individuals reporting greater awareness and attention to personal health selected fewer unhealthy options on the FLVR task. Finally, the FLVR task correlated negatively with self-report of sweat-producing physical activity $(r=-.23) .^{5}$ This suggests that individuals who are more engaged in health-related activity were less likely to make unhealthy selections on the FLVR task.

Conscientiousness was the only personality variable significantly related to the FLVR task $(r=-.30)$. Individuals higher in conscientiousness selected fewer unhealthy food options on target trials. The FLVR task was not correlated with any other measured personality trait, although the alphas for openness and agreeableness were low. The FLVR task was not correlated with mood or BMI. 


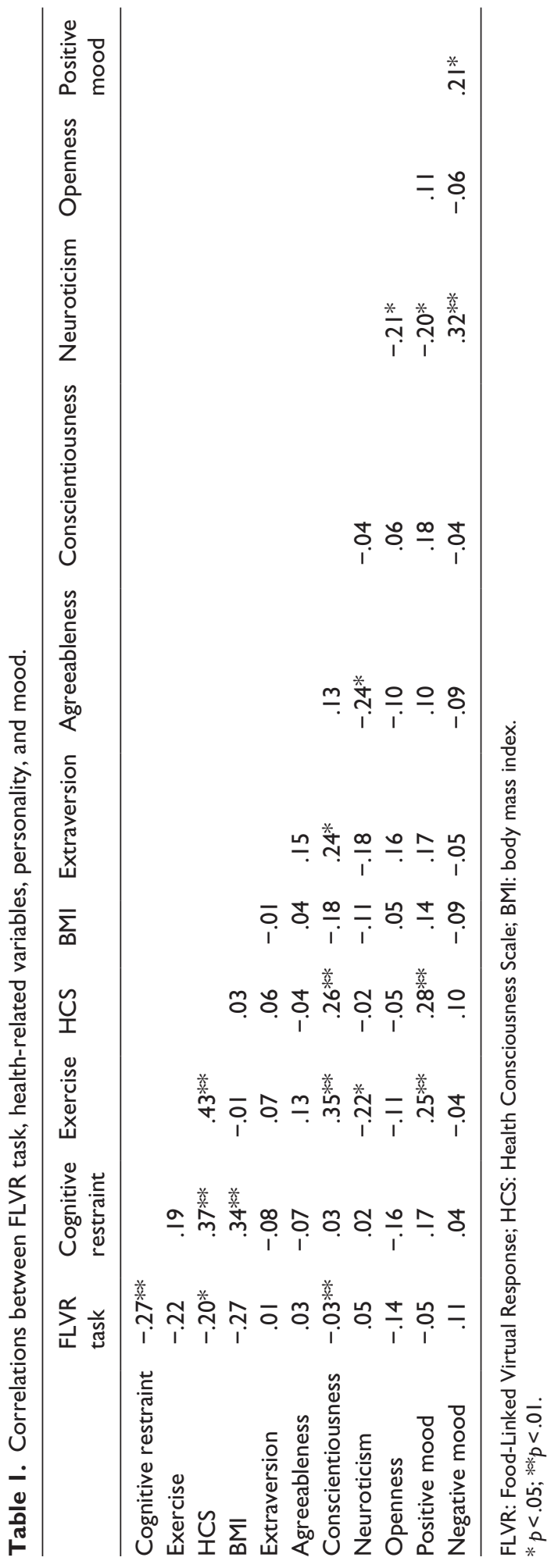


Results suggest that the FLVR task was appropriately correlated with constructs related to dietary decision-making and was unrelated to constructs inconsistently related to dietary decision-making, demonstrating convergent and divergent validity. BMI was unrelated to FLVR task scores, suggesting that the task measures state, not trait, level food decisions. Study 2 was designed to assess predictive validity by determining whether the FLVR task could predict actual food-related selection.

\section{Study 2}

Predictive validity of the FLVR task was examined by assessing its capability for predicting actual dietary choices. The number of unhealthy choices selected on FLVR task target trials was expected to be predictive of the proportion of unhealthy to healthy food selected from a buffet.

\section{Method}

Participants. In total, 80 community members (60\% female; $36.3 \%$ Black, $10 \%$ Asian, $37.5 \%$ White, 5\% Latino, 8.8\% Multiracial, and 2.5\% other; mean age $=32.58$ years, $S D=13.27$ ) were recruited at a psychology laboratory in an urban area of the Southeastern United States.

Procedure. Participants were told they would be engaging in a "food marketing study." Following informed consent, participants completed the FLVR task, other relevant questionnaires, and demographic questions via computer. Upon completion, participants entered a room containing a table of actual food items. Participants were instructed to help themselves to whatever they wanted to be eaten as a snack during the remainder of the study. Participants were left unaccompanied in the room to make their selections. When finished, they took their food back to their computers and completed a final questionnaire before being prompted to list everything they had eaten that day prior to the study. Participants were allowed to eat their chosen snacks during administration of this questionnaire. Upon completion, participants were debriefed regarding the true nature of the study and paid US\$9.

Questionnaires and measures. Participants completed the FLVR task and questionnaires described in Study 1. Participants self-reported height and weight for BMI calculation as well as demographic information.

In-person food validation. Participants were given a plate and led to a room with tables set up with food. Each table arrangement contained 4 sets of 12 food or beverage items ( $1 / 4$ navel orange wedge, $1 / 4$ cup $M \& M^{\circledR}{ }^{\circledR}$ candies, 6 baby carrots, 3 Chips Ahoy ${ }^{\circledR}$ cookies, 6 Doritos ${ }^{\circledR}$ chips, 9 oz water bottle, 6 grapes, 1/4 cup Skittles ${ }^{\circledR}$ candies, 4 slices of celery of $2^{\prime \prime}, 3$ Oreo $^{\circledR}$ cookies, 6 Lays ${ }^{\circledR}$ potato chips, and $9 \mathrm{oz}$ CocaCola $^{\circledR}$ canned soft drink) in varying configurations. All food items were placed in clear $9 \mathrm{oz}$ cups (units). Participants were instructed to take as many cups as they wanted from the table to eat as a snack while they completed the second portion of the study, but that they could not take food home with them. Research assistants noted the number and type of food units selected.

Prior consumption. Participants were asked to list the foods eaten that day. Two independent raters assessed whether participants had eaten a large amount of food with respect to the time of day participation took place. This variable accounted for satiation levels at time of study participation.

\section{Results and discussion}

Three variables assessed actual food selection: total number of units selected (total items), the proportion of unhealthy units selected (unhealthy proportion), and total calories selected (calories). The number of units each participant selected was counted, summed by type (healthy vs unhealthy), and summed together (total items). Proportion of unhealthy units was calculated from the separated sum scores (unhealthy proportion). Calories were 
assessed by estimating the estimated number of calories in each unit based on serving size and summing these calories for each participant.

Table set-up. The units were displayed in one of four arrangements: healthy foods (left), unhealthy foods (right); healthy foods (right), unhealthy foods (left); and two settings with healthy and unhealthy food randomly assorted. Table display was changed after 20 participants. Table arrangement was unrelated to the actual food selection variables suggesting that participants' choices were not influenced by the table placement of healthy and unhealthy foods. Table set-up was not included as a variable in further analyses.

Main analyses. Participants made fewer unhealthy than healthy selections on FLVR task target trials $(M=20.05, S D=11.93)$. On average, participants selected between 4 and 5 units, approximately 340 calories, from the buffet table ( $M$ total units $=4.65, S D=1.71 ; M$ calories $=339.88, S D=211.44)$. Although all participants took at least 1 unit from the table, the amount of calories selected varied, with some participants selecting only units with no calories (water) (range $=1-9$ items). Relatively equal amounts of healthy and unhealthy units were selected $(M$ total number healthy $=2.39$, $S D=1.36 ; M$ total number unhealthy $=2.25$, $S D=1.61)$. Proportionally, slightly more healthy food was selected than unhealthy food, mirroring the mean tally of the FLVR task ( $M$ unhealthy proportion $=.47, S D=.25$ ).

Independent sample $t$-tests examined whether there were group difference on the FLVR task performance. Men and women performed similarly on the FLVR task $(p>.05)$ as did vegetarians and non-vegetarians $(p>.05)$. There were significant differences between racial and ethnic groups for FLVR task performance $(F(5,73)=2.39, p<.05)$, selection of a greater unhealthy proportion of food units $(F(5,73)=9.20, p<.01)$, and number of calories $(F(5,73)=5.77, p<.01)$. Total number of units was not found to differ by race $(p>05)$. Tukey's post hoc tests revealed Black participants $(n=29)$ selected more unhealthy options in the
FLVR task (mean difference $=10.21, p<.02)$, a greater proportion of unhealthy food (mean difference $=.34, p<.01)$, and a greater number of calories (mean difference $=252.60, p<.01)$ than did White participants $(n=30)$. Black participants selected a greater proportion of unhealthy food (mean difference $=.27, p<.02$ ) than did Latino participants $(n=3)$ although the small sample size for Latinos should be noted. No other significant race differences on outcome variables were found. The FLVR task and actual food selection were not correlated with age.

Regression analyses determined whether the FLVR task was predictive of actual food selection independent of other variables associated with dietary habits. Health consciousness was included due to its correlation with the predictor (FLVR task $r=-.54, p<.01$ ) and outcome variables (unhealthy proportion $r=-.52, p<.01$; calories $r=-.421, p<.01)$ and to determine whether the FLVR task was predictive of food selection independently. Prior consumption was included as a covariate. Gender, age, and race/ ethnicity were also included due to their frequent inclusion in diet and health-related studies. $^{6}$ Race/ethnicity was dummy-coded with White as the comparison category.

The FLVR task was a significant predictor of the proportion of unhealthy to healthy units which participants selected $(\beta=.37, t(68)=3.96$, $p<.01$; see Table 2$){ }^{7}$ Individuals selecting more unhealthy options on FLVR task target trials tended to choose a higher proportion of unhealthy food from the buffet than those who selected fewer unhealthy options on the FLVR task. The total number of calories selected was also predicted by the FLVR task $(\beta=.29, t(68)=2.53$, $p<.02)$. Individuals who selected more unhealthy options on FLVR task target trials selected more estimated calories from the buffet.

The FLVR task did not predict the total number of units selected from the buffet table $(p>.05)$. This suggests that the FLVR task is predictive of quality, not quantity, of food selection.

Known groups analyses. To investigate the FLVR task's ability to predict food selection for individuals of varying body sizes, BMI was coded 
Table 2. Regression statistics for FLVR task on unhealthy proportion, calories and total items.

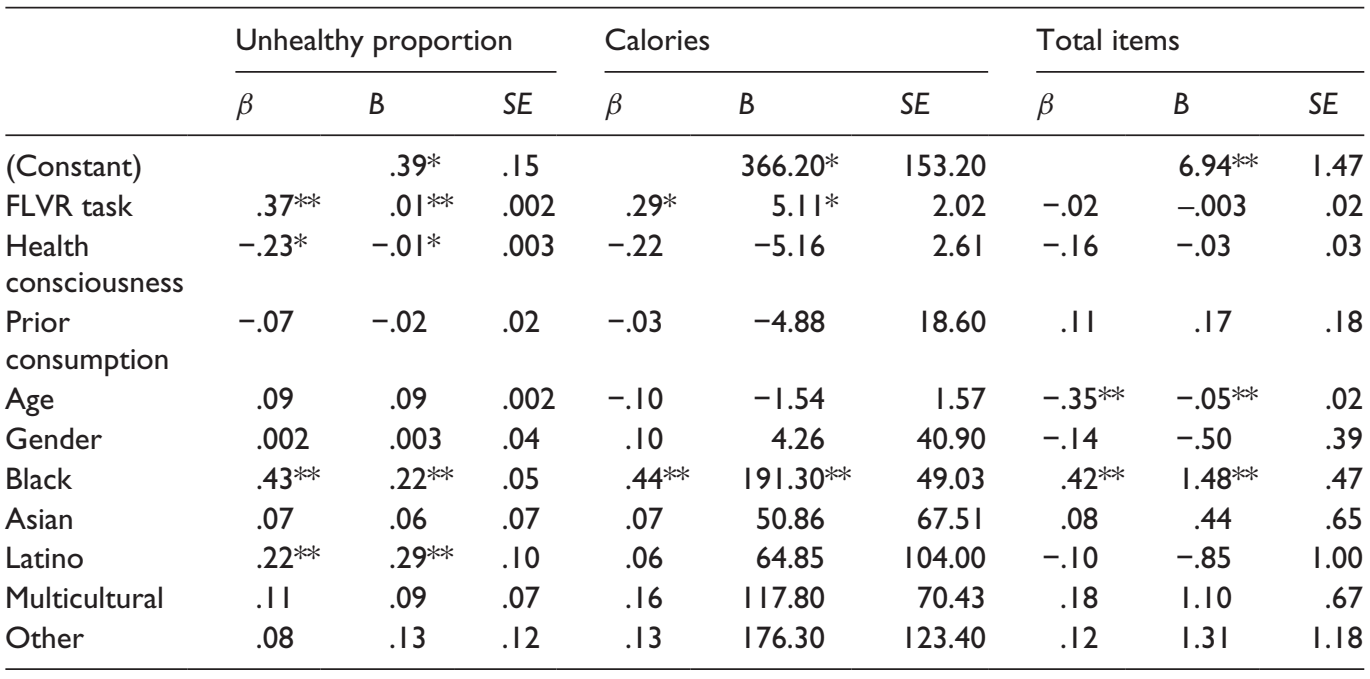

SE: standard error; FLVR: Food-Linked Virtual Response.

$* p<.05 ; * * p<.01$.

into four dummy variables representing established BMI categories (World Health Organization (WHO), 1995). ${ }^{8}$ Interaction variables for these variables and the FLVR task were entered into linear regression models predicting total units, calories, and unhealthy proportion. Only the model for unhealthy proportion showed a significant relationship between BMI category and food selection. Obese individuals selected a greater proportion of unhealthy units $(\beta=.23$, $t(73)=2.29, p<.03)$.

Results suggest that although obese individuals were more likely to choose unhealthy units than non-obese individuals, the FLVR task was predictive of the proportion of unhealthy to healthy units selected by individuals of all BMI ranges. Furthermore, the FLVR task accounted for variability in food selection independent of other variables frequently associated with or controlled for in dietary and health-related studies. Thus, the FLVR task may be a valid measure of momentary food and beverage choice, rather than a measure of health-related personality factors or traits.

Because significant differences existed between Black and White participants on the FLVR task, unhealthy proportion of units, calories, and total units, the relationship between FLVR task and actual food selection for Black $(n=29)$ and White $(n=30)$ individuals was probed. Increased endorsement of unhealthy options on the FLVR task was significantly correlated with the selection of more calories (Black $r=.44$, $p<.02$; White $r=.56, p<.02)$ and a higher proportion of unhealthy units (Black $r=.38, p<.04$; White $r=.77, p<.01$ ) for both groups. This was true for Asians $(n=8$; calories $r=.83, p<.02$; unhealthy proportion $r=.79, p<.03$ ) but not for Latinos $(n=3 ; p s>.05)$, although the low sample size should be noted. FLVR task was not predictive of total units selected for any racial/ethnic group, consistent with the main analysis. This suggests that the FLVR task is predictive of actual food choices across racial/ ethnic groups.

\section{General discussion}

The FLVR task was associated with food selection independent of health-related personality traits and BMI and thus has potential for a variety of applications. Because the FLVR task may be adept at measuring food decisions at the state rather than trait decision level, it may be used as 
an outcome measure in experimental studies measuring how certain factors may change an individual's immediate eating choices. For example, Pascoe and Richman (2010) found that individuals who were asked to reflect upon a discriminatory event were more likely to select a greater number of unhealthy options on the prototype version of the FLVR task than did individuals who reflected on daily habits. A follow-up study revealed that participants who were experimentally subjected to negative and discriminatory evaluation selected more unhealthy options on the FLVR task prototype target trials than did participants who were subjected to negative but ostensibly fair evaluation.

The FLVR task is also flexible in its scoring. Although originally developed to highlight unhealthy decisions, the task can be easily scored to represent healthy choices by summing healthy choices on target trials rather than unhealthy choices. This scoring does not change the results of the study except to reverse the valence of the regression coefficient for the FLVR task, allowing for interpretations that are consistent with the framing of the research question.

The FLVR task is also predictive of food choice across racial/ethnic groups. While Black participants were more likely to select unhealthy units than Whites, the FLVR task was predictive of overall outcomes for both groups. The predictive ability of the FLVR task for other racial/ethnic groups is less certain due to small sample size. Future research might address predictive ability for these groups.

Inconsistency in the correlation between FLVR task scores and age across studies may be due to the restricted age range found in Study 1 (college-aged participants) whereby Study 2 was more representative of a community sample. While this is a factor that may warrant more exploration, as a whole, the FLVR task seems to perform well in predicting food choice in an adult population.

There may be additional personality variables related to FLVR performance that were not assessed. Further testing of the FLVR task could correlate with personality factors other than conscientiousness. Also, although it provides an opportunity for researchers to assess quality of dietary choice in an easy, inexpensive format, the FLVR task is still limited in its predictive ability. It cannot be used to predict the total number of items a person might eat, but rather the proportional quality of food likely to be selected. Additionally, it is unknown whether the FLVR task can account for actual intake of food. This study did not systematically monitor whether participants actually ate all selected food, although non-systematic observation suggests that the majority of participants consumed what they had taken. Moreover, a recent study using a buffet-style task similar to the one used in this study found that the majority of individuals (Study 1: $88 \%$ and Study 2: 100\%) consumed all of their self-chosen food (Robinson et al., 2014).

\section{Conclusion}

The FLVR task was found to be psychometrically sound and was associated with quality of momentary dietary choice. These results support the future use of this measure to examine how situational factors may impact the expected quality of dietary decision-making.

\section{Funding}

This research received no specific grant from any funding agency in the public, commercial, or not-forprofit sectors.

\section{Notes}

1. A table containing the target trial food pairings can be found in the Supplementary Material for this article.

2. Means and standard deviations (SDs) of Study 1 and Study 2 variables are available in the Supplementary Material for this article.

3. Nine participants $(8.4 \%)$ reported a vegetarian diet.

4. Two participants were excluded from analyses due to unlikely self-reported BMI measurements of 13.95 and 5.15 (reported height of $5^{\prime}$ $11^{\prime \prime}$ and weight of 100 pounds and height of 5' $4 "$ and weight of 30 pounds, respectively).

5. Because of the variability with which participants answered the Godin Leisure-Time Exercise Questionnaire (GLTEQ) (e.g. adding 
total minutes, counting number of 15 -minute blocks, and counting number of activities lasting longer than 15 minutes), data were unreliable. Only the final question of the GLTEQ was used in analysis.

6. Additional regression models excluding health consciousness and prior consumption but including age, ethnicity, and gender revealed no reduction in the ability of the Food-Linked Virtual Response (FLVR) task to predict food selections and are not reported in the text.

7. One participant was excluded from analyses on the basis of reporting an unlikely body mass index (BMI) of 12.7 (reported height of $6^{\prime} 5^{\prime \prime}$ and weight of 110 pounds).

8. Underweight $(n=3)$, healthy weight $(n=36)$, overweight $(n=16)$, and obese $(n=23)$.

\section{References}

Bogg T and Roberts BW (2004) Conscientiousness and health-related behaviors: A meta-analysis of the leading behavioral contributors to mortality. Psychological Bulletin 130(6): 887-919.

Brogan A and Hevey D (2013) Eating styles in the morbidly obese: Restraint eating, but not emotional and external eating, predicts dietary behaviour. Psychology \& Health 28(6): 714-725.

Godin G and Shepherd RJ (1997) Godin LeisureTime Exercise Questionnaire. Medicine and Science in Sports and Exercise 29(Suppl): S36-S38.

Gould SJ (1988) Consumer attitudes toward health and health care: A differential perspective. Journal of Consumer Affairs 22(1): 96-118.
Kern ML and Friedman HS (2008) Do conscientious individuals live longer? A quantitative review. Health Psychology 27(5): 505-512.

Koball AM, Meers MR, Storfer-Isser A, et al. (2012) Eating when bored: Revision of the emotional eating scale with a focus on boredom. Health Psychology 31(4): 521-524.

Lee RE, McNeill L, O'Connor DP, et al (2011) Multiple measures of physical activity dietary habits and weight status in African American and Hispanic or Latina women. Journal of Community Health 36(6): 1011-1023.

Pascoe EA and Richman LS (2010) Effect of discrimination on food decisions. Self and Identity 10(3): 396-406.

Rammstedt B and John O (2007) Measuring personality in one minute or less: A 10-item short version of the big five inventory in English and German. Journal of Research in Personality 41(1): 203-212.

Robinson E, Fleming A and Higgs S (2014) Prompting healthier eating: Testing the use of health and social norm based messages. Health Psychology 33(9): 1057-1064.

Stunkard AJ and Messick S (1985) The three-factor eating questionnaire to measure dietary restraint, disinhibition and hunger. Journal of Psychosomatic Research 29(1): 71-83.

Watson D, Clark LA and Tellegen A (1988) Development and validation of brief measures of positive and negative affect: The PANAS scales. Journal of Personality and Social Psychology 54(6): 1063-1070.

World Health Organization (WHO) (1995) Physical status: The use and interpretation of anthropometry. Report of a WHO Expert Committee, WHO, Geneva. 